Conflict of interest: None declared.

\title{
References
}

1 Haahtela T, Lindholm H, Björksten F, et al. The prevalence of asthma in Finnish young men. BMJ 1990; 301: 266-268.

2 Asher MI, Montefort S, Björkstén B, et al. Worldwide time trends in the prevalence of symptoms of asthma, allergic rhinoconjunctivitis, and eczema in childhood: ISAAC Phases One and Three repeat multicountry cross-sectional surveys. Lancet 2006; 368: 733-743.

3 Ege MJ, Mayer M, Normand AC, et al. Exposure to environmental microorganisms and childhood asthma. $N$ Engl J Med 2011; 364: 701-709.

4 Haahtela T, Laatikainen T, Alenius $\mathrm{H}$, et al. Hunt for the origin of allergy - comparing the Finnish and Russian Karelia. Clin Exp Allergy 2015; 45: 891-901.

5 Borodulin K, Vartiainen E, Peltonen M, et al. Forty-year trends in cardiovascular risk factors in Finland. Eur J Public Health 2015; 25: 539-546.

6 Burney PG, Luczynska C, Chinn S, et al. The European Community Respiratory Health Survey. Eur Respir J 1994; 7: 954-960.

7 Vartiainen E, Petäys T, Haahtela T, et al. Allergic diseases, skin prick test responses, and IgG levels in North Karelia, Finland, and the Republic of Karelia, Russia. J Allergy Clin Immunol 2002; 109: 643-648.

8 Laatikainen T, von Hertzen L, Koskinen JP, et al. Allergy gap between Finnish and Russian Karelia on increase. Allergy 2011; 66: 886-892.

9 von Mutius E, Fritzsch C, Weiland SK, et al. Prevalence of asthma and allergic disorders among children in united Germany: a descriptive comparison. BMJ 1992; 305: 1395-1399.

10 Riikjärv MA, Julge K, Vasar M, et al. The prevalence of atopic sensitization and respiratory symptoms among Estonian schoolchildren. Clin Exp Allergy 1995; 25: 1198-1204.

11 Viinanen A, Munhbayarlah S, Zevgee T, et al. Prevalence of asthma, allergic rhinoconjunctivitis and allergic sensitization in Mongolia. Allergy 2005; 60: 1370-1377.

12 Haahtela T, Holgate ST, Pawankar R, et al. The biodiversity hypothesis and allergic disease: world allergy organization position statement. World Allergy Organ J 2013; 6: 3.

13 Hesla HM, Stenius F, Jäderlund L, et al. Impact of lifestyle on the gut microbiota of healthy infants and their mothers - the ALADDIN birth cohort. FEMS Microbiol Ecol 2014; 90: 791-801.

14 Haahtela T, von Hertzen L, Mäkelä M, et al. Finnish Allergy Programme 2008-2018 - time to act and change the course. Allergy 2008; 63: 634-645.

\section{Obstructive sleep apnoea as a risk factor for osteopenia and osteoporosis in the male population}

To the Editor:

Obstructive sleep apnoea (OSA) is a sleep disorder characterised by recurrent apnoea events leading to hypoxia, hypercapnia and sleep disruption [1]. OSA represents a growing health problem mainly affecting men; in fact, its prevalence in the adult male population is between $4 \%$ and $24 \%$ [1-3].

It was recently demonstrated that OSA may lead to a deficient vitamin D status inducing a secondary hyperparathyroidism, which may produce the demineralisation of the skeleton and a reduction of the bone mineral density (BMD) [3]. In keeping with this observation, recent studies documented reduced BMD in OSA patients, thus speculating that OSA may represent a risk factor for bone resorption [4-6]. However, the evidence proposed so far is controversial due to differences in patient populations, study designs and definition of OSA; principally, since physical activity and body mass index (BMI) can negatively influence $\mathrm{BMD}$, major limitations of the previous studies are the lack of physical activity assessment and absence of BMI-matched controls $[7,8]$.

Therefore, the aim of the present study was to evaluate bone homeostasis in a large cohort of male OSA patients compared to male controls matched for age, BMI and physical activity, also investigating and correlating the measured BMD to polygraphic parameters, Epworth Sleepiness Scale (ESS) scores and serum biomarker levels, such as vitamin $\mathrm{D}$, parathormone $(\mathrm{PTH})$, calcium, fibrinogen and C-reactive protein (CRP). 
We screened 240 consecutive, male, severe OSA (apnoea-hypopnea index $>30$ per h) patients undergoing polygraphic cardiorespiratory monitoring from October 2014 to March 2015; 148 patients were excluded and 92 patients (mean \pm SD age $51.17 \pm 11.82$ years, BMI $30.90 \pm 5.87 \mathrm{~kg} \cdot \mathrm{m}^{-2}$ and ESS score $10.73 \pm 6.03$ ) were included in the study. We compared the OSA population to a sample of 50 controls (age 51.00 \pm 11.68 years, BMI $30.78 \pm 1.93 \mathrm{~kg} \cdot \mathrm{m}^{-2}$ and ESS score $5.67 \pm 2.29$ ) matched for age and BMI with the OSA patients. Exclusion criteria for OSA patients and controls were: concomitant neurological and/or psychiatric diseases; chronic liver disease or chronic renal failure; chronic obstructive pulmonary disease; diabetes; thyroid dysfunction; malignancies; use of corticosteroids or antibiotics over the 4 weeks preceding recruitment in the study; autoimmune disorders; symptoms or signs of acute or chronic inflammatory disorders or recent infections; calcium or vitamin D supplements; diuretic treatments; heavy smoking; alcohol abuse; hypogonadism; and a history of immobilisation or fractures.

All patients and controls underwent: demographic, medical history and medication assessment; a venous blood sample between 08:00 and 09:00 h after overnight fasting; and dual-energy X-ray absorptiometry (DEXA) measurement of BMD in the lumbar spine and femur. DEXA results are expressed as absolute BMD values and as T-scores. T-score evaluates how the examined value is different from that of the standard population (healthy subjects of the same sex at the bone mass peak). We referred to "osteopenia" when the T-score value was $<-1 \mathrm{SD}$ and to "osteoporosis" when it was $<-2.5 \mathrm{SD}$ [9].

Using Student's t-test to compare data between groups, we documented that OSA patients showed significantly lower vitamin D $\left(18.62 \pm 8.02\right.$ versus $\left.31.64 \pm 15.03 \mathrm{ng} \cdot \mathrm{mL}^{-1}, \mathrm{p}<0.0001\right)$, and higher PTH $(62.74 \pm 23.63$ versus $\left.54.32 \pm 12.19 \mathrm{pg} \cdot \mathrm{mL}^{-1}, \mathrm{p}<0.05\right)$, fibrinogen $\left(404.41 \pm 101.89\right.$ versus $\left.316.74 \pm 43.72 \mathrm{mg} \cdot \mathrm{dL}^{-1}, \mathrm{p}<0.001\right)$ and CRP serum levels $\left(3.69 \pm 4.29\right.$ versus $\left.1.82 \pm 0.97 \mathrm{mg} \cdot \mathrm{L}^{-1}, \mathrm{p}<0.001\right)$ than controls. We found a significant reduction of $\mathrm{BMD}$ in all the regions analysed of both the lumbar spine and femur in OSA patients with respect to controls (figure 1a). Moreover, Chi-squared analysis with correction for continuity showed that T-scores consistent with osteopenia/osteoporosis were more frequent in OSA patients than controls at all regions of the lumbar spine, and at the femur neck, upper femur neck and Ward triangle (figure $1 \mathrm{~b}$ ).

Finally, we performed a functional evaluation of performance status and physical activity using the Physical Activity Scale for the Elderly (PASE) test, which is a brief, easily scored, reliable and valid instrument for the assessment of physical activity in young-old populations over a 1-week period [10]. On the basis of PASE test scores, OSA patients and controls did not differ in terms of physical activity $(83.90 \pm 35.41$ versus $86.38 \pm 35.86)$.

The Pearson correlation test documented significant correlations between lower BMD in the lumbar spine and femur, lower mean arterial oxygen saturation $\left(\mathrm{SaO}_{2}\right)$ and $\mathrm{SaO}_{2}$ nadir, and higher time spent with an $\mathrm{SaO}_{2}<90 \%$. Moreover, lower BMD in several lumbar spine and femur regions also correlated with higher ESS scores and higher BMI.

Therefore, this study documented that male OSA patients are affected by reduced BMD in the lumbar spine and femur, thus suffering more frequently from osteopenia and osteoporosis in those regions with respect to age-, BMI- and physical activity-matched male controls. Since BMD reduction in several lumbar and femur

a)

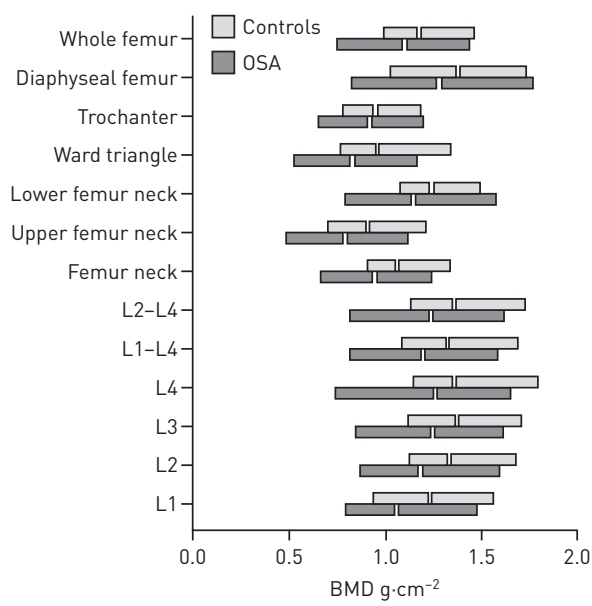

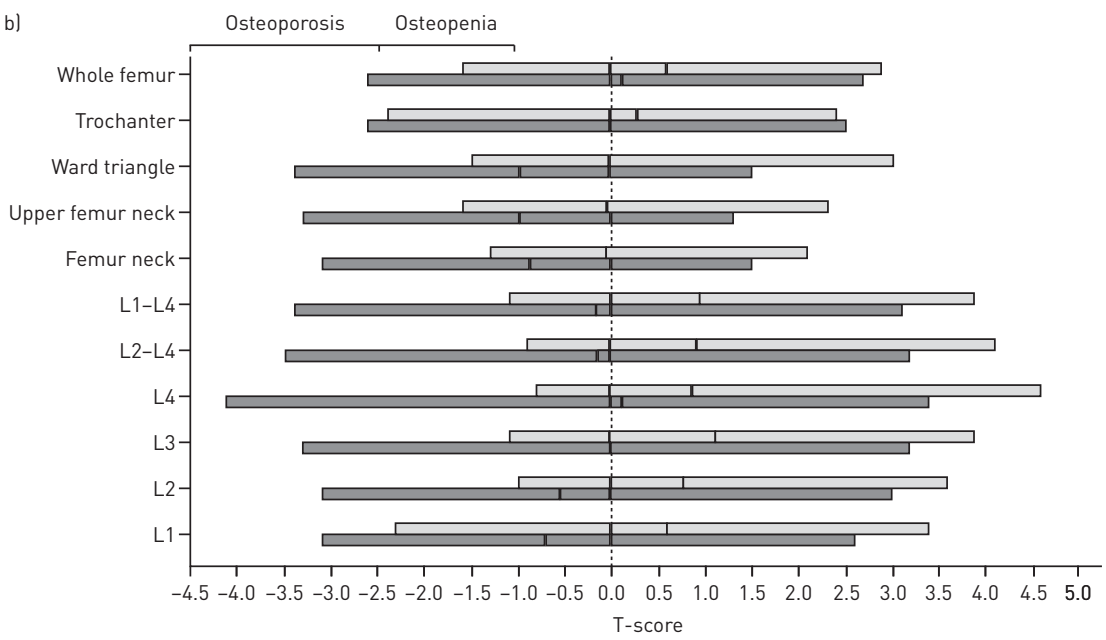

FIGURE 1 a) Significant reduction of bone mineral density (BMD) in all the lumbar spine (L) and femur regions of obstructive sleep apnoea (OSA) patients compared to controls ( $p<0.05$ for trochanter; $p<0.001$ for all other regions). b) Comparison between T-scores of OSA patients and controls, which is significant at all regions of the lumbar spine, and at the femur neck, upper femur neck and Ward triangle ( $p<0.01$ for all regions). 
segments significantly correlated with the alteration of night oxygen saturation indices, hypoxia seems to be the main candidate in reducing BMD in male severe OSA patients. In agreement with this observation, hypoxia has been closely related to changes in bone turnover, and recent in vitro studies have shown that hypoxia promotes osteoclast formation and activity whereas inhibits osteoblast function, thus determining bone resorption $[11,12]$. Indeed, we hypothesised that lower nocturnal oxygen levels, a characteristic of OSA syndrome, could be responsible for the reduction of BMD in OSA patients, which results in osteopenia/osteoporosis.

However, the aetiology of bone resorption, and then osteopenia/osteoporosis, in OSA patients could be complex and multifactorial, also including alterations in vitamin D homeostasis, chronic systemic inflammation, and reduced physical activity related to sleepiness and obesity. Accordingly, we documented vitamin D insufficiency coupled with secondary hyperparathyroidism and increased systemic inflammation, characterised by higher CRP and fibrinogen levels, in OSA patients. Nevertheless, the lack of correlations between BMD and serum biomarker data does not propose a reciprocal link among systemic chronic inflammation, alteration in vitamin D status and bone metabolism derangement in OSA patients. Furthermore, since OSA patients and control population did not differ in terms of physical activity, age and BMI, we can exclude insufficient physical activity, obesity and ageing as putative factors of reduced BMD in OSA patients. Although reduction of BMD could also be influenced by the disease duration, sleep disordered breathing can occur without awareness, thus making difficult the quantification of disease duration [13].

The absence of an interventional treatment, such as positive airway pressure (PAP), is the major limitation of this study, since we cannot test the possible restorative effect of PAP therapy on BMD in OSA patients.

Finally, although we are aware that osteoporosis is more frequent in women [14], we did not include female OSA patients in this study as they already show changes in sexual hormones due to post-menopausal condition, which primary influences bone turnover and metabolism, and because prevalence of OSA in women is low. Hence, considering that osteoporosis is a growing concern in the male population, in which a more complete picture of osteoporosis prevalence and aetiopathogenesis is needed, we selectively conducted this study in men.

In conclusion, taking into account that bone diseases are rarely considered in the evaluation of OSA patients, this report proposes the clinical potential of performing DEXA in male patients, since OSA could be a detrimental factor on BMD leading to osteopenia and osteoporosis, and thus giving susceptibility to bone fractures. On these bases, we encourage clinicians to assess BMD especially in male OSA patients, in consideration of the possibility of starting early interventions to reduce the future risk of fracture.

0 @ERSpublications

This report proposes the clinical potential of monitoring bone mineral density in male OSA patients http://ow.ly/Voe47

Claudio Liguori ${ }^{1}$, Nicola Biagio Mercuri ${ }^{1,2,3}$, Francesca Izzi $^{1}$, Andrea Romigi ${ }^{1}$, Alberto Cordella ${ }^{2}$, Eleonora Piccirilli ${ }^{4}$, Salvatore Viola ${ }^{4}$, Silvio Costa ${ }^{5}$, Paolo Sbraccia ${ }^{5}$, Maria Grazia Marciani ${ }^{3}$, Umberto Tarantino ${ }^{4}$ and Fabio Placidi ${ }^{1}$

${ }^{1}$ Sleep Medicine Centre, Neurophysiopathology Unit, Dept of Systems Medicine, University of Rome "Tor Vergata", Rome, Italy. ${ }^{2}$ Fondazione Santa Lucia IRCCS, Rome, Italy. ${ }^{3}$ Neurology Unit, Dept of Systems Medicine, University of Rome "Tor Vergata", Rome, Italy. ${ }^{4}$ Dept of Orthopaedics and Traumatology, University of Rome "Tor Vergata", Rome, Italy. ${ }^{5}$ Obesity Center, Dept of Systems Medicine, University of Rome "Tor Vergata", Rome, Italy.

Correspondence: Claudio Liguori, Sleep Medicine Centre, Neurophysiopathology Unit, Dept of Systems Medicine, University of Rome "Tor Vergata”, Viale Oxford 81, 00133 Rome, Italy. E-mail: dott.claudioliguori@yahoo.it

Received: July 172015 | Accepted after revision: Nov 182015 | First published online: Jan 072016

Conflict of interest: None declared.

\section{References}

1 Young T, Peppard PE, Gottlieb DJ. Epidemiology of obstructive sleep apnea: a population health perspective. Am J Respir Crit Care Med 2002; 165: 1217-1239.

2 Placidi F, Diomedi M, Cupini LM, et al. Impairment of daytime cerebrovascular reactivity in patients with obstructive sleep apnoea syndrome. J Sleep Res 1998; 7: 288-292.

3 Liguori C, Romigi A, Izzi F, et al. Continuous positive airway pressure treatment increases serum vitamin D levels in male patients with obstructive sleep apnea. J Clin Sleep Med 2015; 11: 603-607.

4 Uzkeser $\mathrm{H}$, Yildirim K, Aktan B, et al. Bone mineral density in patients with obstructive sleep apnea syndrome. Sleep Breath 2013; 17: 339-342.

5 Chen YL Weng SF, Shen YC, et al. Obstructive sleep apnea and risk of osteoporosis: a population-based cohort study in Taiwan. J Clin Endocrinol Metab 2014; 99: 2441-2447.

6 Terzi R, Yllmaz Z. Bone mineral density and changes in bone metabolism in patients with obstructive sleep apnea syndrome. J Bone Miner Metab 2015 [In press DOI: 10.1007/s00774-015-0691-1]. 
7 Rosen CJ. The epidemiology and pathogenesis of osteoporosis. In: De Groot LJ, Beck-Peccoz P, Chrousos G, et al., eds. Endotext. South Dartmouth, MDText.com Inc., 2000.

8 Chilibeck PD, Sale DG, Webber CE. Exercise and bone mineral density. Sports Med 1995; 19: $103-122$.

9 Orimo H, Hayashi Y, Fukunaga M, et al. Diagnostic criteria for primary osteoporosis: year 2000 revision. J Bone Miner Metab 2001; 19: 331-337.

10 Washburn RA, McAuley E, Katula J, et al. The Physical Activity Scale for the Elderly (PASE): evidence for validity. J Clin Epidemiol 1999; 52: 643-651.

11 Arnett TR, Gibbons DC, Utting JC, et al. Hypoxia is a major stimulator of osteoclast formation and bone resorption. J Cell Physiol 2003; 196: 2-8.

12 Utting JC, Robins SP, Brandao-Burch A, et al. Hypoxia inhibits the growth, differentiation and bone-forming capacity of rat osteoblasts. Exp Cell Res 2006; 312: 1693-1702.

13 Gooneratne NS, Vitiello MV. Sleep in older adults: normative changes, sleep disorders, and treatment option. Clin Geriatr Med 2014; 30: 591-627.

14 McClain JJ, Lewin DS, Laposky AD, et al. Associations between physical activity, sedentary time, sleep duration and daytime sleepiness in US adults. Prev Med 2014; 66: 68-73.

\section{Deficient interleukin-17 production in response to Mycobacterium abscessus in cystic fibrosis}

To the Editor:

The respiratory tract of patients with cystic fibrosis (CF) is colonised with a high diversity of micro-organisms. Nontuberculous mycobacteria (NTM) show a high and increasing prevalence. $40 \%$ of these positive NTM cultures are caused by Mycobacterium abscessus [1], one of the rapidly growing NTMs present in the environment. Patients with M. abscessus infection are difficult to treat, due to natural and acquired antibiotic resistance $[2,3]$, and an infection with $M$. abscessus is controversially discussed as a contraindication for lung transplantation [4].

Immune-modulatory treatment strategies might contribute to overcome this problem. For their development, a better understanding of the defective immune response explaining the higher susceptibility of CF patients to M. abscessus is needed. Here we present three CF patients with M. abscessus infection, in whom we describe the pathogen-specific innate and adaptive cytokine production and compare this with non-CF patients with pulmonary infection caused by various NTMs: M. abscessus $(\mathrm{n}=1)$, M. avium $(\mathrm{n}=3)$, M. kansasii $(\mathrm{n}=2)$ and M. intracellulare $(\mathrm{n}=1)$.

Case 1 is a 24 -year-old female patient with CF (dF508del/dF508del) with pancreatic insufficiency and Pseudomonas colonisation since 2003. In 2004, she presented with allergic bronchopulmonary aspergillosis (ABPA) which was successfully treated with corticosteroids. After years of infectious exacerbations she presented with an episode of haemoptysis in 2010. Shortly thereafter, M. abscessus was cultured from her sputum. In 2011, haemoptysis and clinical deterioration led to hospitalisation and several courses of antimycobacterial regimens (combinations of amikacin, clarithromycin, tigecycline, meropenem and clofazimine) were given without successful M. abscessus eradication.

Case 2 is a 23-year-old male patient with CF (dF508del/dF508del), pancreatic insufficiency and Staphylococcus aureus and Aspergillus colonisation who presented with ABPA. After a course of corticosteroids and itraconazole, he improved and serological markers for ABPA have remained at low levels ever since. After this episode, M. abscessus was consistently cultured and although he had no physical complaints, his pulmonary function deteriorated, and a computed tomography thorax scan showed several subpleural and intraparenchymatous nodular lesions compatible with mycobacterial disease. No clearance of $M$. abscessus was achieved, despite two courses of treatment with combination regimens of amikacin, meropenem, clarithromycin and clofazimine.

Case 3 is a 15-year-old male patient with CF (dF508del/G551D), pancreatic insufficiency and Pseudomonas colonisation. Since 2011 M. abscessus has been consistently cultured and he experienced several exacerbations in 2014, in which his pulmonary function deteriorated, despite several NTM regimens (including tigecycline, 